\title{
Approximation by special values of harmonic zeta function and log-sine integrals
}

\author{
EMRE ALKAN
}

\begin{abstract}
Motivated by applications of log-sine integrals to a wide range of mathematical and physical problems, it is shown that real numbers and certain types of log-sine integrals can be strongly approximated by linear combinations of special values of the harmonic zeta function with the property that the coefficients belonging to these combinations turn out to be universal in the sense of being independent of special values. The approximation of real numbers by combinations of special values is reminiscent of the classical Diophantine approximation of Liouville numbers by rationals. Moreover, explicit representations of some specific log-sine integrals are obtained in terms of special values of the harmonic zeta function and the Riemann zeta function through a study of Fourier series involving harmonic numbers. In particular, special values of the harmonic zeta function and the less studied odd harmonic zeta function are expressed in terms of log-sine integrals over $[0,2 \pi]$ and $[0, \pi]$.
\end{abstract}

\section{Introduction}

Log-sine integrals have applications to a wide range of mathematical and physical problems. To quote a basic example of how they arise in physics, consider two charged particles placed on the unit circle. If both particles carry a unit charge, then the potential energy of the system is

$$
-\log \left|1-\mathrm{e}^{\mathrm{i} t}\right|=-\log \left(2 \sin \frac{t}{2}\right)
$$

where $0<t<2 \pi$ is the relative angle between the particles. Consequently, the average value of this potential energy on the unit circle leads to the 
convergent improper integral

$$
-\frac{1}{2 \pi} \int_{0}^{2 \pi} \log \left(2 \sin \frac{t}{2}\right) d t,
$$

which is the simplest prototype of a log-sine integral and is known to vanish. Similar integrals arising from more general configurations of freely moving charges on a thin circular conductor of unit radius were studied by Dyson and Mehta (see [33-37] and Chapter 16 of [47]). Their insight led to the following partition integral describing the charge distribution:

$$
\frac{1}{(2 \pi)^{n}} \int_{-\pi}^{\pi} \ldots \int_{-\pi}^{\pi} \prod_{1 \leq j<k \leq n}\left|\mathrm{e}^{\mathrm{i} \theta_{j}}-\mathrm{e}^{\mathrm{i} \theta_{k}}\right| d \theta_{1} \cdots d \theta_{n} .
$$

This integral turns out to be a special case of a family of integrals introduced and evaluated by Selberg [52] in terms of values of the gamma function and more recently by Aomoto [13] who gave a simpler approach to a slightly more general integral (see also Chapter 8 of [11] and Chapter 17 of [47]). For a detailed historical account of the Selberg integral, we recommend the article by Forrester and Warnaar [38]. Log-sine integrals of the form

$$
\int_{0}^{2 \pi} t^{q} \log ^{p}\left(2 \sin \frac{t}{2}\right) d t
$$

for nonnegative integers $p, q$ have occupied a central place in recent literature. They were evaluated in many different ways (see [24-27]), have deeper connections to values of polylogarithms $[21,44,45]$ and rapidly converging series representations of $L$-values, a subject that goes back to Euler and Ramanujan (see [7,41] and Chapter 14 of [17]). Improving on the work of Shen [53], Choi and Srivastava [27] found an algorithm for the evaluation of log-sine (and log-cosine) integrals, which essentially reduces this computation to successive differentiations of a function of two variables depending on the digamma function. In this way, they have tabulated their results for certain ranges of $p$ and $q$. In this paper, we show that strong approximations of real numbers and log-sine integrals are possible via special values of the harmonic zeta function and the Riemann zeta function defined respectively by

$$
\zeta_{H}(s)=\sum_{n=1}^{\infty} \frac{H_{n}}{n^{s}} \text { and } \zeta(s)=\sum_{n=1}^{\infty} \frac{1}{n^{s}}
$$


for $\mathfrak{R}(s)>1$, where

$$
H_{n}=\sum_{k=1}^{n} \frac{1}{k}
$$

is the $n$th harmonic number. Special values of the Riemann zeta function play an increasingly sophisticated role in arithmetic and $K$-theory. Euler's classical formula on even values, namely

$$
\zeta(2 m)=(-1)^{m-1} \frac{(2 \pi)^{2 m}}{2(2 m) !} B_{2 m}
$$

where $B_{2 m}$ are the (nonvanishing) Bernoulli numbers for $m \geq 1$, unexpectedly comes up in some energy calculations belonging to quantum mechanics and in Bose condensation phenomena of statistical quantum mechanics (see p. 38 and p. 243 of [39]). On the other hand, the elusive odd values $\zeta(2 m+1)$ are described by Bloch [18], Kato [43] and Bloch and Kato [19] by building a new theory of Tamagawa measure on motives. All of these insights are now collected under the far reaching Beilinson conjectures [50] on the arithmetic nature of special values of $L$-functions. Further important links between special values, multiple values, Mahler measures and Boyd's conjectures can be found in the works of Boyd [22], Ohno and Zudilin [49] and Rogers and Zudilin [51]. For other perspectives on the structure of $L$-values, the reader is referred to the works of Andrews et al. [12] and the author [2-4,6]. Since harmonic numbers lack any obvious multiplicative structure, $\zeta_{H}$ does not have an Euler product and the theory of the harmonic zeta function is more limited in comparison with that of the Riemann zeta function. Despite this drawback, special values of the harmonic zeta function have surprising relations to those of the Riemann zeta function. Concerning this, Euler obtained the elegant formula (see p. 252 of [16])

$$
2 \zeta_{H}(m)=(m+2) \zeta(m+1)-\sum_{k=1}^{m-2} \zeta(m-k) \zeta(k+1)
$$

for all $m \geq 2$, where the sum over $k$ is taken to be zero when $m=2$. The particular cases

$$
\sum_{n=1}^{\infty} \frac{H_{n}}{n^{2}}=2 \zeta(3) \text { and } \sum_{n=1}^{\infty} \frac{H_{n}}{n^{3}}=\frac{5}{4} \zeta(4)=\frac{\pi^{4}}{72}
$$

of Euler's formula are indeed striking. There is also a rich collection of infinite series identities involving harmonic numbers. We refer to the papers of 
De Doelder [32], D. Borwein and J. M. Borwein [20], Chu [28,29] and Chen and Chu [23] for different approaches to obtaining such identities. Hyperharmonic numbers, which are generalizations of harmonic numbers, were first introduced by Conway and Guy [30]. The combinatorial significance of these numbers was investigated by Benjamin et al. [15]. Further combinatorial connections and relations between values of the hyperharmonic zeta function and the Hurwitz zeta function were nicely treated by Mezö and Dil [48]. Our main result below shows that specific log-sine type integrals and consequently all real numbers can be strongly approximated by linear combinations of even values of the harmonic zeta function teamed up by the odd values of the Riemann zeta function. The surprising feature of these combinations is the independence of coefficients from special values. Furthermore, the error in these approximations tends to zero as the number of terms in the combinations tends to infinity and this is analogous to what happens in a typical Diophantine approximation result, namely that the error tends to zero as the height of the approximating number tends to infinity, where in case of rational approximating numbers, the height essentially corresponds to the denominator. We should mention that approximation type results in some nonstandard settings have been the subject of important research. The monograph of Karatsuba and Voronin (see Chapter 7 of [42]) contains many beautiful results on how close one can get to the values of analytic functions using shifted values of $L$-functions. Functional independence of very general families of $L$-functions (such as the ones that are associated to cusp forms) was obtained by Zaharescu and Zaki [54] with the help of a purely algebraic method. The author [5] showed that weighted averages of Gauss sums can be well approximated by $\mathbb{Q}$-linear combinations of special values of $L$-functions in which coefficients belonging to the combinations only depend on the parity of the character. Alkan et al. $[9,10]$ found some nonstandard Diophantine approximation results with values of arithmetic functions and Fourier coefficients of newforms. It is worth remarking that our results below on the approximation of real numbers with combinations of special values were discovered only through a study of log-sine integrals so were motivated from physics, and it is reasonable to ask if there is a direct approach to such results bypassing the role of log-sine integrals.

If $F$ is a nonempty set of real numbers (in this paper $F$ is always taken to be a vector space or a field or its multiplicative group in $\mathbb{R}$ ), then an $F$-linear combination of real numbers $x_{1}, \ldots, x_{n}$ will be written as

$$
\sum_{k=1}^{n} F x_{k}
$$


unless it is necessary to distinguish the actual coefficients. As usual, $\mathbb{Q}(\pi)$ denotes the totally transcendental extension obtained by adjoining $\pi$ to $\mathbb{Q}$. Equivalently, it is the field of evaluations of all $\mathbb{Q}$-rational functions at $\pi$. Let us write $\mathbb{Q}\left[\pi^{s}: 1 \leq s \leq n\right]$ to denote the vector space of all $\mathbb{Q}$-linear combinations of $\pi^{s}$ for $1 \leq s \leq n$. We are now ready to state our main result.

Theorem 1. Let $f$ be a Lipschitz continuous function defined on $[0,2 \pi]$ that is not a polynomial. Then the integral

$$
I_{f, 2 \pi}:=\int_{0}^{2 \pi} f(t) \log \left(2 \sin \frac{t}{2}\right) d t
$$

exists and for any given integer $n \geq 3$, there exists an integer $r \leq n$ tending to infinity with $n$ and having the property that if $r \geq 2$, then there is a linear combination $\mathcal{L}$ of the form

$$
\mathcal{L}=\sum_{k=1}^{[r / 2]} \mathbb{Q}\left[\pi^{s}: 1 \leq s \leq r-2 k+1\right]\left(\zeta_{H}(2 k)-\zeta(2 k+1)\right)
$$

whose coefficients are all nonzero, depend on $f$ and $n$ but not on the collection of values $\zeta_{H}(2 k)-\zeta(2 k+1), \quad k \geq 1$, satisfying

$$
\left|I_{f, 2 \pi}-\mathcal{L}\right| \ll_{f} \frac{1}{n}
$$

Moreover, if $f$ is a $\mathcal{C}^{\infty}$ function defined on $[0,2 \pi]$ that is not a polynomial, then for any integer $q \geq 1$, there are such combinations whose coefficients are all nonzero, depend on $f, n$ and $q$ but not on the collection of values $\zeta_{H}(2 k)-\zeta(2 k+1), \quad k \geq 1$, satisfying

$$
\left|I_{f, 2 \pi}-\mathcal{L}\right| \ll_{f, q} \frac{1}{n^{q}}
$$

when $r \geq 2$. Consequently, if $\alpha$ is any real number, then there are such combinations whose coefficients are all nonzero, depend on $\alpha, n$ and $q$ but not on the collection of values $\zeta_{H}(2 k)-\zeta(2 k+1), \quad k \geq 1$, satisfying

$$
|\alpha-\mathcal{L}| \ll_{\alpha, q} \frac{1}{n^{q}}
$$

when $r \geq 2$. 
The set of numbers of the form $\mathcal{L}$ in Theorem 1 is countable. Thus, we conclude that for almost all real numbers $\alpha$ (in the sense of Lebesgue measure),

$$
0<|\alpha-\mathcal{L}| \ll_{\alpha, q} \frac{1}{n^{q}}
$$

when $r \geq 2$. This is reminiscent of the classical Diophantine approximation of Liouville numbers by rationals. Recall that a real number $\alpha$ is a Liouville number, if for any integer $q \geq 1$, there exists a rational number $\frac{m}{n}$ with $n>1$ satisfying

$$
0<\left|\alpha-\frac{m}{n}\right|<\frac{1}{n^{q}} .
$$

Liouville numbers are the first examples of transcendental numbers and belong to the set of $U$-numbers according to Mahler's classification (see [46]). Concerning this, the author [8] recently discovered new series and families of transcendental numbers that are not $U$-numbers represented by these series through a study of integral representations involving carefully selected rational functions whose evaluations lead to specific linear combinations with algebraic coefficients of $\pi$ and Baker periods. It is possible to make strong approximations using odd values of the harmonic zeta function as well at the cost of introducing a rational polynomial evaluated at $\pi$.

Theorem 2. Let $f$ be a Lipschitz continuous function defined on $[0,2 \pi]$ that is not a polynomial. Then the integral

$$
J_{f, 2 \pi}:=\int_{0}^{2 \pi} f(t) \log ^{2}\left(2 \sin \frac{t}{2}\right) d t
$$

exists and for any given integer $n \geq 3$, there exists an integer $r \leq n$ tending to infinity with $n$ and a polynomial $P_{r}(x) \in \mathbb{Q}[x]$ of degree $r$ with coefficients depending only on $f$ and $n$ such that if $r \geq 2$, then there is a linear combination $\mathcal{K}$ of the form

$$
\mathcal{K}=\sum_{k=1}^{[r / 2]} \mathbb{Q}\left[\pi^{s}: 1 \leq s \leq r-2 k+1\right] \zeta_{H}(2 k+1),
$$

whose coefficients are all nonzero, depend on $f$ and $n$ but not on the collection of values $\zeta_{H}(2 k+1), k \geq 1$, satisfying

$$
\left|J_{f, 2 \pi}-\left(\pi^{3} P_{r}(\pi)+\mathcal{K}\right)\right| \ll_{f} \frac{1}{n} .
$$


If $f$ is a $\mathcal{C}^{\infty}$ function defined on $[0,2 \pi]$ that is not a polynomial, then for any integer $q \geq 1$, there are such combinations whose coefficients are all nonzero, depend on $f, n$ and $q$ but not on the collection of values $\zeta_{H}(2 k+1), k \geq 1, a$ polynomial $P_{r}(x) \in \mathbb{Q}[x]$ of degree $r$ with coefficients depending only on $f, n$ and $q$, satisfying

$$
\left|J_{f, 2 \pi}-\left(\pi^{3} P_{r}(\pi)+\mathcal{K}\right)\right| \ll_{f, q} \frac{1}{n^{q}}
$$

when $r \geq 2$. Consequently, if $\alpha$ is any real number, then there are such combinations whose coefficients are all nonzero, depend on $\alpha, n$ and $q$ but not on the collection of values $\zeta_{H}(2 k+1), \quad k \geq 1$, a polynomial $P_{r}(x) \in \mathbb{Q}[x]$ of degree $r$ with coefficients depending only on $\alpha, n$ and $q$, satisfying

$$
\left|\alpha-\left(\pi^{3} P_{r}(\pi)+\mathcal{K}\right)\right| \ll_{\alpha, q} \frac{1}{n^{q}}
$$

when $r \geq 2$.

\section{Explicit representations of some log-sine integrals}

For the proofs of our main results, we first need to obtain explicit representations of the log-sine moments

$$
I_{m, a}:=\int_{0}^{a} t^{m} \log \left(2 \sin \frac{t}{2}\right) d t \text { and } J_{m, a}:=\int_{0}^{a} t^{m} \log ^{2}\left(2 \sin \frac{t}{2}\right) d t
$$

where $m$ is a positive integer and $0<a \leq 2 \pi$, especially when $a=2 \pi$. Let us define an odd harmonic number as

$$
O_{n}:=\sum_{k=1}^{n} \frac{1}{2 k-1}
$$

for $n \geq 1$. Then the odd harmonic zeta function is given by

$$
\zeta_{O}(s)=\sum_{n=1}^{\infty} \frac{O_{n}}{n^{s}}
$$

for $\mathfrak{R}(s)>1$. Special values of $\zeta_{O}$ are less understood and not so easily accessible as special values of $\zeta_{H}$. Despite this, one still has the curious relation (see [20])

$$
\zeta_{O}(2)=\frac{7}{4} \zeta(3)
$$


The values $\zeta_{O}(m)$ for integers $m \geq 3$ do not directly admit any closed form evaluations in terms of other well-known constants. Nevertheless, in the last section, we give one such formula for $\zeta_{O}(3)$ along with explicit computations of some log-sine integrals over $[0, \pi]$. Our last result in this section shows that $\zeta_{O}(m)$ can always be written as a combination of log-sine integrals over $[0,2 \pi]$ and $[0, \pi]$.

Theorem 3. If $m \geq 1$, then

$$
\begin{aligned}
& I_{m+1,2 \pi}-\pi I_{m, 2 \pi} \\
& \quad=\sum_{k=1}^{[(m+1) / 2]}(-1)^{k} \frac{2 m !}{(m-2 k+2) !}(2 \pi)^{m-2 k+2}\left(\zeta_{H}(2 k)-\zeta(2 k+1)\right) .
\end{aligned}
$$

Consequently,

$$
\zeta_{H}(m+1)-\zeta(m+2)=\sum_{\substack{2 \leq k \leq m+2 \\ k \equiv \sigma}} \mathbb{Q}(\pi) I_{k, 2 \pi}
$$

when $m \geq 1$ is odd and $\sigma=0,1$. The coefficients belonging to $\mathbb{Q}(\pi)$ in this combination are all computable.

Theorem 4. If $m \geq 2$, then

$$
\begin{aligned}
J_{m, 2 \pi}= & -\frac{2^{m-1}\left(m^{2}-7 m-6\right)}{3(m+1)(m+2)(m+3)} \pi^{m+3} \\
& +\sum_{k=1}^{[m / 2]}(-1)^{k-1} \frac{2 m !}{(m-2 k+1) !}(2 \pi)^{m-2 k+1} \zeta_{H}(2 k+1) .
\end{aligned}
$$

Consequently,

$$
\zeta_{H}(m+1)=\mathbb{Q} \pi^{m+2}+\sum_{\substack{2 \leq k \leq m+1 \\ k \equiv \sigma}} \mathbb{Q}(\pi) J_{k, 2 \pi}
$$

when $m \geq 2$ is even and $\sigma=0,1$. The coefficients belonging to this combination are all computable. 
Concerning values of the odd harmonic zeta function, we have

Theorem 5. If $m \geq 3$ is odd, then

$$
\zeta_{O}(m)=\sum_{n=1}^{\infty} \frac{O_{n}}{n^{m}}=\mathbb{Q} \pi^{m+1}+\sum_{k=1}^{(m-1) / 2} \mathbb{Q}(\pi) J_{2 k+1,2 \pi}+\sum_{k=1}^{m-1} \mathbb{Q}(\pi) J_{k, \pi}
$$

and if $m \geq 2$ is even, then

$$
\zeta_{O}(m)=\sum_{k=1}^{m / 2} \mathbb{Q}(\pi) I_{2 k+1,2 \pi}+\sum_{k=1}^{m} \mathbb{Q}(\pi) I_{k, \pi}
$$

The coefficients belonging to these combinations are all computable.

\section{Proof of Theorem 3}

We know that for $|z|=1$ and $z \neq 1$,

$$
\sum_{n=1}^{\infty}\left(\frac{H_{n}}{n}-\frac{1}{n^{2}}\right) z^{n}=\frac{1}{2} \log ^{2}(1-z),
$$

where the logarithm has its principal value. Taking $z=e^{i t}$ for $0<t<2 \pi$ in (3.1) and then comparing the imaginary parts of both sides, one obtains the Fourier sine series of the function $\frac{1}{2}(t-\pi) \log \left(2 \sin \frac{t}{2}\right)$ in the form

$$
\sum_{n=1}^{\infty}\left(\frac{H_{n}}{n}-\frac{1}{n^{2}}\right) \sin n t=\frac{1}{2}(t-\pi) \log \left(2 \sin \frac{t}{2}\right)
$$

for $0<t<2 \pi$. For $k \geq 1$, consider the partial sums

$$
\sum_{n=1}^{k} \sin n t=\frac{\sin \frac{k t}{2} \sin \frac{(k+1) t}{2}}{\sin \frac{t}{2}}
$$

Assume that $t \in[\delta, 2 \pi-\delta]$, where $\delta>0$ is fixed but arbitrary. For these values of $t$, it follows from (3.3) that

$$
\left|\sum_{n=1}^{k} \sin n t\right|=O_{\delta}(1)
$$


Thus, by (3.4), the partial sums are uniformly bounded in terms of $\delta$. Moreover,

$$
\frac{H_{n}}{n}-\frac{1}{n^{2}}=\frac{H_{n-1}}{n}
$$

(with the convention $H_{0}=0$ ) is monotonically decreasing to zero for $n \geq 2$ and by Dirichlet's criterion, the Fourier series

$$
\sum_{n=1}^{\infty}\left(\frac{H_{n}}{n}-\frac{1}{n^{2}}\right) \sin n t
$$

converges uniformly on $[\delta, 2 \pi-\delta]$. For any integer $m \geq 1$, multiplying both sides of (3.2) by $t^{m}$, then integrating from $\delta$ to $2 \pi-\delta$ and noticing that the left-hand side can be integrated term by term as a result of uniform convergence on $[\delta, 2 \pi-\delta]$, we deduce that

$$
\sum_{n=1}^{\infty}\left(\frac{H_{n}}{n}-\frac{1}{n^{2}}\right) \int_{\delta}^{2 \pi-\delta} t^{m} \sin n t d t=\frac{1}{2} \int_{\delta}^{2 \pi-\delta} t^{m}(t-\pi) \log \left(2 \sin \frac{t}{2}\right) d t
$$

for $\delta>0$. Define

$$
a_{m}:=\int_{\delta}^{2 \pi-\delta} t^{m} \sin n t d t
$$

By partial integration, one easily derives from (3.6) the recursive formula

$$
\begin{aligned}
a_{m}= & \frac{1}{n}\left(\delta^{m} \cos n \delta-(2 \pi-\delta)^{m} \cos n(2 \pi-\delta)\right) \\
& +\frac{m}{n^{2}}\left((2 \pi-\delta)^{m-1} \sin n(2 \pi-\delta)-\delta^{m-1} \sin n \delta\right)-\frac{m(m-1)}{n^{2}} a_{m-2}
\end{aligned}
$$

Combining (3.5)-(3.7), we have

$$
\begin{aligned}
& \sum_{n=1}^{\infty}\left(\frac{H_{n}}{n}-\frac{1}{n^{2}}\right) \int_{\delta}^{2 \pi-\delta} t^{m} \sin n t d t \\
& \quad=\sum_{n=1}^{\infty}\left(\frac{H_{n}}{n^{2}}-\frac{1}{n^{3}}\right)\left(\delta^{m} \cos n \delta-(2 \pi-\delta)^{m} \cos n(2 \pi-\delta)\right)
\end{aligned}
$$




$$
\begin{aligned}
& +m \sum_{n=1}^{\infty}\left(\frac{H_{n}}{n^{3}}-\frac{1}{n^{4}}\right)\left((2 \pi-\delta)^{m-1} \sin n(2 \pi-\delta)-\delta^{m-1} \sin n \delta\right) \\
& -m(m-1) \sum_{n=1}^{\infty}\left(\frac{H_{n}}{n^{3}}-\frac{1}{n^{4}}\right) a_{m-2} .
\end{aligned}
$$

Letting $\delta \rightarrow 0^{+}$and using the fact that all of the resulting series are uniformly convergent with respect to $\delta$, we see from (3.8) that

$$
\begin{aligned}
& \lim _{\delta \rightarrow 0^{+}} \sum_{n=1}^{\infty}\left(\frac{H_{n}}{n}-\frac{1}{n^{2}}\right) \int_{\delta}^{2 \pi-\delta} t^{m} \sin n t d t \\
& \quad=-(2 \pi)^{m}\left(\zeta_{H}(2)-\zeta(3)\right)-m(m-1) \lim _{\delta \rightarrow 0^{+}} \sum_{n=1}^{\infty}\left(\frac{H_{n}}{n^{3}}-\frac{1}{n^{4}}\right) a_{m-2} .
\end{aligned}
$$

We may now iterate $(3.9)$ and apply the same reasoning to $a_{m-2}, a_{m-4}, \ldots$ and then let $\delta \rightarrow 0^{+}$. Clearly, this process terminates after finitely many iterations. On the other hand, the improper integral on the right-hand side of (3.5) converges as a result of the mild logarithmic singularity, and recalling the definition $(2.1)$ of $I_{m, 2 \pi}$, one obtains

$$
\lim _{\delta \rightarrow 0^{+}} \int_{\delta}^{2 \pi-\delta} t^{m}(t-\pi) \log \left(2 \sin \frac{t}{2}\right) d t=I_{m+1,2 \pi}-\pi I_{m, 2 \pi}
$$

for $m \geq 1$. In particular, integrating

$$
\sum_{n=1}^{\infty}\left(\frac{H_{n}}{n}-\frac{1}{n^{2}}\right) t^{m} \sin n t
$$

term by term from 0 to $2 \pi$ is justified. Some caution is necessary to keep track of the end of this iteration process. Because of the recursion (3.7) satisfied by $a_{m}$, the final term can indeed be specified in terms of the parity of $m$. Precisely, using (3.10), we may write

$$
\sum_{n=1}^{\infty}\left(\frac{H_{n}}{n}-\frac{1}{n^{2}}\right) \int_{0}^{2 \pi} t^{m} \sin n t d t=\frac{1}{2}\left(I_{m+1,2 \pi}-\pi I_{m, 2 \pi}\right) .
$$

Noting that

$$
\int_{0}^{2 \pi} t^{m} \sin n t d t=\sum_{k=1}^{[(m+1) / 2]}(-1)^{k} \frac{m !(2 \pi)^{m-2 k+2}}{(m-2 k+2) ! n^{2 k-1}}
$$


when $m \geq 1$, we infer from (3.11) and (3.12) that

$$
\begin{aligned}
& I_{m+1,2 \pi}-\pi I_{m, 2 \pi} \\
& \quad=\sum_{k=1}^{[(m+1) / 2]}(-1)^{k} \frac{2 m !}{(m-2 k+2) !}(2 \pi)^{m-2 k+2}\left(\zeta_{H}(2 k)-\zeta(2 k+1)\right)
\end{aligned}
$$

when $m \geq 1$. Let us now show by induction over odd $m \geq 1$ that

$$
\zeta_{H}(m+1)-\zeta(m+2)=\sum_{\substack{2 \leq k \leq m+2 \\ k \equiv 0}} \mathbb{Q}(\pi) I_{k, 2 \pi}=\sum_{k=1}^{(m+1) / 2} \mathbb{Q}(\pi) I_{2 k, 2 \pi}
$$

holds. When $m=1$, we have $\zeta_{H}(2)-\zeta(3)=\zeta(3)$ and $I_{2,2 \pi}=-4 \pi \zeta(3)$ (see [27]) so that this settles the base case by taking the coefficient $-\frac{1}{4 \pi} \in$ $\mathbb{Q}(\pi)$. Since $m-1$ is even, we may rewrite (3.13) as

$$
\begin{aligned}
& I_{m, 2 \pi}-\pi I_{m-1,2 \pi} \\
& \quad=\sum_{k=1}^{(m-1) / 2}(-1)^{k} \frac{2(m-1) !}{(m-2 k+1) !}(2 \pi)^{m-2 k+1}\left(\zeta_{H}(2 k)-\zeta(2 k+1)\right) .
\end{aligned}
$$

Combining (3.13) and (3.14), we see that

$$
\begin{aligned}
& I_{m+1,2 \pi}-\pi^{2} I_{m-1,2 \pi}=(-1)^{\frac{m+1}{2}} 4 m ! \pi\left(\zeta_{H}(m+1)-\zeta(m+2)\right) \\
& \quad+\sum_{k=1}^{(m-1) / 2}(-1)^{k}\left(\frac{2 m !}{(m-2 k+2) !}+\frac{(m-1) !}{(m-2 k+1) !}\right)(2 \pi)^{m-2 k+2} \\
& \quad \times\left(\zeta_{H}(2 k)-\zeta(2 k+1)\right) .
\end{aligned}
$$

Next using the inductive hypothesis, one has

$$
\zeta_{H}(2 k)-\zeta(2 k+1)=\sum_{j=1}^{k} \mathbb{Q}(\pi) I_{2 j, 2 \pi}
$$


for $1 \leq k \leq \frac{m-1}{2}$. Feeding (3.16) into (3.15), it follows that

$$
\begin{aligned}
& (-1)^{\frac{m+1}{2}} 4 m ! \pi\left(\zeta_{H}(m+1)-\zeta(m+2)\right)=I_{m+1,2 \pi}-\pi^{2} I_{m-1,2 \pi} \\
& -\sum_{k=1}^{(m-1) / 2}(-1)^{k}\left(\frac{2 m !}{(m-2 k+2) !}+\frac{(m-1) !}{(m-2 k+1) !}\right)(2 \pi)^{m-2 k+2} \\
& \quad \times\left(\sum_{j=1}^{k} \mathbb{Q}(\pi) I_{2 j, 2 \pi}\right)=I_{m+1,2 \pi}-\pi^{2} I_{m-1,2 \pi}+\sum_{j=1}^{(m-1) / 2} \mathbb{Q}(\pi) I_{2 j, 2 \pi} \\
& =\sum_{j=1}^{(m+1) / 2} \mathbb{Q}(\pi) I_{2 j, 2 \pi} .
\end{aligned}
$$

Clearly, (3.17) gives that

$$
\zeta_{H}(m+1)-\zeta(m+2)=\sum_{j=1}^{(m+1) / 2} \mathbb{Q}(\pi) I_{2 j, 2 \pi}
$$

and this completes the induction for odd $m \geq 1$. The induction step also shows that if the $\mathbb{Q}(\pi)$ coefficients of $\zeta_{H}(2 k)-\zeta(2 k+1)$ for $1 \leq k \leq \frac{m-1}{2}$ are all computable, then so are the $\mathbb{Q}(\pi)$ coefficients of $\zeta_{H}(m+1)-\zeta(m+2)$ via (3.15). Similarly, to show

$$
\zeta_{H}(m)-\zeta(m+1)=\sum_{\substack{2 \leq k \leq m+2 \\ k \equiv 1}} \mathbb{Q}(\pi) I_{k, 2 \pi}=\sum_{k=1}^{m / 2} \mathbb{Q}(\pi) I_{2 k+1,2 \pi}
$$

by induction over even $m \geq 2$, first note that the base case $m=2$ holds by $I_{3,2 \pi}=-12 \pi^{2} \zeta(3)$ (see [27]). Then use (3.13) to complete the induction step. The computability of all coefficients in $\mathbb{Q}(\pi)$ again follows from the induction step. This completes the proof of Theorem 3.

\section{Proof of Theorem 4}

Let us rewrite (3.1) as

$$
\sum_{n=1}^{\infty} \frac{H_{n}}{n} z^{n}=\frac{1}{2} \log ^{2}(1-z)+\operatorname{Li}_{2}(z)
$$


for $|z|=1, z \neq 1$, where

$$
\operatorname{Li}_{2}(z)=\sum_{n=1}^{\infty} \frac{z^{n}}{n^{2}}
$$

is the dilogarithm. Taking $z=\mathrm{e}^{\mathrm{i} t}$ for $0<t<2 \pi$ in (4.1) and then comparing real parts, we arrive at the Fourier cosine series

$$
\sum_{n=1}^{\infty} \frac{H_{n}}{n} \cos n t=\frac{1}{2} \log ^{2}\left(2 \sin \frac{t}{2}\right)-\frac{(t-\pi)^{2}}{8}+\mathfrak{R}\left(\operatorname{Li}_{2}\left(\mathrm{e}^{\mathrm{i} t}\right)\right)
$$

where $\mathfrak{R}\left(\mathrm{Li}_{2}\left(\mathrm{e}^{\mathrm{i} t}\right)\right)=\mathrm{Gl}_{2}\left(\mathrm{e}^{\mathrm{i} t}\right)$ is known as Clausen's $\mathrm{Gl}_{2}$-function. It is known that (see [45, Equation (2.49)])

$$
\mathrm{Gl}_{2}\left(\mathrm{e}^{\mathrm{i} t}\right)=\frac{\pi^{2}}{6}-\frac{t(2 \pi-t)}{4}
$$

for $0 \leq t \leq 2 \pi$ (see also the work of Albeverio and Cacciapuoti [1] for new representations of the Riemann zeta function in terms of the Mellin transform of $\mathrm{Gl}_{2}$ ). From (4.2) and (4.3), the formula

$$
\sum_{n=1}^{\infty} \frac{H_{n}}{n} \cos n t=\frac{\pi^{2}}{24}-\frac{\pi t}{4}+\frac{t^{2}}{8}+\frac{1}{2} \log ^{2}\left(2 \sin \frac{t}{2}\right)
$$

is verified for $0<t<2 \pi$. As in the proof of Theorem 3, we can show that the Fourier cosine series on the left-hand side of (4.4) is uniformly convergent on any interval of form $[\delta, 2 \pi-\delta]$, where $\delta>0$ is fixed but arbitrary. Integrating termwise, (4.4) gives that

$$
\begin{aligned}
& \sum_{n=1}^{\infty} \frac{H_{n}}{n} \int_{\delta}^{2 \pi-\delta} t^{m} \cos n t d t \\
& \quad=\int_{\delta}^{2 \pi-\delta}\left(\frac{\pi^{2}}{24}-\frac{\pi t}{4}+\frac{t^{2}}{8}\right) t^{m} d t+\frac{1}{2} \int_{\delta}^{2 \pi-\delta} t^{m} \log ^{2}\left(2 \sin \frac{t}{2}\right) d t
\end{aligned}
$$

for $m \geq 1$. By partial integration,

$$
\begin{aligned}
b_{m}:= & \int_{\delta}^{2 \pi-\delta} t^{m} \cos n t d t=\frac{1}{n}\left((2 \pi-\delta)^{m} \sin n(2 \pi-\delta)-\delta^{m} \sin n \delta\right) \\
& +\frac{m}{n^{2}}\left((2 \pi-\delta)^{m-1} \cos n(2 \pi-\delta)-\delta^{m-1} \cos n \delta\right)-\frac{m(m-1)}{n^{2}} b_{m-2}
\end{aligned}
$$


By (4.6), left-hand side of (4.5) equals

$$
\begin{aligned}
& \sum_{n=1}^{\infty} \frac{H_{n}}{n^{2}}\left((2 \pi-\delta)^{m} \sin n(2 \pi-\delta)-\delta^{m} \sin n \delta\right) \\
& \quad+m \sum_{n=1}^{\infty} \frac{H_{n}}{n^{3}}\left((2 \pi-\delta)^{m-1} \cos n(2 \pi-\delta)\right. \\
& \left.\quad-\delta^{m-1} \cos n \delta\right)-m(m-1) \sum_{n=1}^{\infty} \frac{H_{n}}{n^{3}} b_{m-2}
\end{aligned}
$$

Using the fact that the resulting series in (4.7) are all uniformly convergent in $\delta$, we may let $\delta \rightarrow 0^{+}$and iterate (4.7) to justify that

$$
\sum_{n=1}^{\infty} \frac{H_{n}}{n} t^{m} \cos n t
$$

can be integrated term by term from 0 to $2 \pi$. Letting $\delta \rightarrow 0^{+}$and referring to the evaluation

$$
\int_{0}^{2 \pi} t^{m} \cos n t d t=\sum_{k=1}^{[m / 2]}(-1)^{k-1} \frac{m !(2 \pi)^{m-2 k+1}}{(m-2 k+1) ! n^{2 k}}
$$

for $m \geq 2$, one can easily verify from (4.4) the formula,

$$
\begin{aligned}
J_{m, 2 \pi}= & -\frac{2^{m-1}\left(m^{2}-7 m-6\right)}{3(m+1)(m+2)(m+3)} \pi^{m+3} \\
& +\sum_{k=1}^{[m / 2]}(-1)^{k-1} \frac{2 m !(2 \pi)^{m-2 k+1}}{(m-2 k+1) !} \zeta_{H}(2 k+1)
\end{aligned}
$$

for $m \geq 2$. Let us show by induction that

$$
\zeta_{H}(m)=\mathbb{Q} \pi^{m+1}+\sum_{k=1}^{(m-1) / 2} \mathbb{Q}(\pi) J_{2 k+1,2 \pi}
$$


for odd $m \geq 3$. The identity $\zeta_{H}(3)=\frac{\pi^{4}}{72}$ (see [20]) settles the base case $m=3$. We may rewrite $(4.8)$ as

$$
\begin{aligned}
J_{m, 2 \pi}= & (-1)^{\frac{m-3}{2}} 4 m ! \pi^{2} \zeta_{H}(m)+\mathbb{Q} \pi^{m+3} \\
& +\sum_{k=1}^{(m-3) / 2}(-1)^{k-1} \frac{2 m !(2 \pi)^{m-2 k+1}}{(m-2 k+1) !} \zeta_{H}(2 k+1) .
\end{aligned}
$$

Using the inductive hypothesis, we have

$$
\zeta_{H}(2 k+1)=\mathbb{Q} \pi^{2 k+2}+\sum_{r=1}^{k} \mathbb{Q}(\pi) J_{2 r+1,2 \pi}
$$

for $1 \leq k \leq \frac{m-3}{2}$. It follows that

$$
\begin{aligned}
& \sum_{k=1}^{(m-3) / 2}(-1)^{k-1} \frac{2 m !(2 \pi)^{m-2 k+1}}{(m-2 k+1) !}\left(\mathbb{Q} \pi^{2 k+2}+\sum_{r=1}^{k} \mathbb{Q}(\pi) J_{2 r+1,2 \pi}\right) \\
& \quad=\mathbb{Q} \pi^{m+3}+\sum_{r=1}^{(m-3) / 2} \mathbb{Q}(\pi) J_{2 r+1,2 \pi} .
\end{aligned}
$$

Induction is then completed by gathering these equations. The induction step and (4.8) further show that all of the coefficients are computable. The proof of the formula

$$
\zeta_{H}(m+1)=\mathbb{Q} \pi^{m+2}+\sum_{k=1}^{m / 2} \mathbb{Q}(\pi) J_{2 k, 2 \pi}
$$

for even $m \geq 2$ and computability of the coefficients follow by a similar induction using (4.8). This completes the proof of Theorem 4.

\section{Proof of Theorem 5}

Adding (4.4) to (4.4) in which $t$ has been replaced by $\pi-t$, one obtains the Fourier cosine series

$$
\sum_{n=1}^{\infty} \frac{H_{2 n}}{n} \cos 2 n t=-\frac{\pi^{2}}{24}-\frac{\pi t}{4}+\frac{t^{2}}{4}+\frac{1}{2} \log ^{2}\left(2 \sin \frac{t}{2}\right)+\frac{1}{2} \log ^{2}\left(2 \cos \frac{t}{2}\right)
$$


for $0<t<\pi$. The partial sums

$$
\sum_{n=1}^{k} \cos 2 n t=\frac{\sin k t \cos (k+1) t}{\sin t}
$$

again satisfy

$$
\left|\sum_{n=1}^{k} \cos 2 n t\right|=O_{\delta}(1)
$$

when $t \in[\delta, \pi-\delta]$, where $\delta>0$ is fixed but arbitrary. Since $\frac{H_{2 n}}{n}$ is monotonic and tending to zero, the Fourier cosine series in (5.1) is uniformly convergent on $[\delta, \pi-\delta]$. Thus as before, integrating the left-hand side of (5.1) term by term from 0 to $\pi$ is justified. In this way, we obtain

$$
\begin{aligned}
& \sum_{n=1}^{\infty} \frac{H_{2 n}}{n} \int_{0}^{\pi} t^{m} \cos 2 n t d t=\int_{0}^{\pi}\left(-\frac{\pi^{2}}{24}-\frac{\pi t}{4}+\frac{t^{2}}{4}\right) t^{m} d t \\
& \quad+\frac{1}{2} \int_{0}^{\pi} t^{m} \log ^{2}\left(2 \sin \frac{t}{2}\right) d t+\frac{1}{2} \int_{0}^{\pi} t^{m} \log ^{2}\left(2 \cos \frac{t}{2}\right) d t
\end{aligned}
$$

for any odd $m \geq 3$. First observe that

$$
\begin{gathered}
\int_{0}^{\pi} t^{m} \cos 2 n t d t=\frac{1}{2^{m+1}} \int_{0}^{2 \pi} t^{m} \cos n t d t \\
\quad=\frac{1}{2^{m+1}} \sum_{k=1}^{(m-1) / 2}(-1)^{k-1} \frac{m !(2 \pi)^{m-2 k+1}}{(m-2 k+1) ! n^{2 k}} .
\end{gathered}
$$

We may also write

$$
\int_{0}^{\pi}\left(-\frac{\pi^{2}}{24}-\frac{\pi t}{4}+\frac{t^{2}}{4}\right) t^{m} d t=\mathbb{Q} \pi^{m+3}
$$

and

$$
\begin{aligned}
& \int_{0}^{\pi} t^{m} \log ^{2}\left(2 \cos \frac{t}{2}\right) d t=\int_{0}^{\pi}(\pi-t)^{m} \log ^{2}\left(2 \sin \frac{t}{2}\right) d t \\
& =\pi^{m} \int_{0}^{\pi} \log ^{2}\left(2 \sin \frac{t}{2}\right) d t+\sum_{k=1}^{m}\left(\begin{array}{c}
m \\
k
\end{array}\right)(-1)^{k} \pi^{m-k} \int_{0}^{\pi} t^{k} \log ^{2}\left(2 \sin \frac{t}{2}\right) d t .
\end{aligned}
$$


Note that the Fourier sine series

$$
\sum_{n=1}^{\infty} \frac{H_{n}}{n^{2}} \sin n t
$$

is uniformly convergent on $[0,2 \pi]$ and the Fourier cosine series

$$
\sum_{n=1}^{\infty} \frac{H_{n}}{n} \cos n t
$$

is uniformly convergent on any interval of form $[\delta, 2 \pi-\delta]$ with $\delta>0$. Thus we obtain from (4.4) that

$$
\sum_{n=1}^{\infty} \frac{H_{n}}{n^{2}} \sin n t=\frac{\pi^{2} t}{24}-\frac{\pi t^{2}}{8}+\frac{t^{3}}{24}+\frac{1}{2} J_{0, t}+C
$$

for $0<t<2 \pi$, where $C$ is a constant. But letting $t \rightarrow 0^{+}$, we see that $C=0$. Now taking $t=\pi$ in (5.6), one has

$$
J_{0, \pi}=\frac{\pi^{3}}{12}
$$

Assembling (5.2)-(5.5) and (5.7), we deduce that

$$
\sum_{k=1}^{(m-1) / 2}(-1)^{k-1} \frac{m !(2 \pi)^{m-2 k+1}}{2^{m+1}(m-2 k+1) !} \sum_{n=1}^{\infty} \frac{H_{2 n}}{n^{2 k+1}}=\mathbb{Q} \pi^{m+3}+\sum_{k=1}^{m-1} \mathbb{Q}(\pi) J_{k, \pi} .
$$

Let us show by induction that

$$
\sum_{n=1}^{\infty} \frac{H_{2 n}}{n^{m}}=\mathbb{Q} \pi^{m+1}+\sum_{k=1}^{m-1} \mathbb{Q}(\pi) J_{k, \pi}
$$

when $m \geq 3$ is odd. The base case $m=3$ is clear since the left-hand side of (5.8) becomes

$$
\frac{3 \pi^{2}}{4} \sum_{n=1}^{\infty} \frac{H_{2 n}}{n^{3}}
$$


Using the inductive hypothesis, we may rewrite the left-hand side of (5.8) as

$$
\begin{aligned}
& \sum_{k=1}^{(m-3) / 2}(-1)^{k-1} \frac{m !(2 \pi)^{m-2 k+1}}{2^{m+1}(m-2 k+1) !}\left(\mathbb{Q} \pi^{2 k+2}+\sum_{r=1}^{2 k} \mathbb{Q}(\pi) J_{r, \pi}\right) \\
& \quad+(-1)^{\frac{m-3}{2}} \frac{m ! \pi^{2}}{2^{m}} \sum_{n=1}^{\infty} \frac{H_{2 n}}{n^{m}}=\mathbb{Q} \pi^{m+3}+\sum_{r=1}^{m-3} \mathbb{Q}(\pi) J_{r, \pi} \\
& \quad+(-1)^{\frac{m-3}{2}} \frac{m ! \pi^{2}}{2^{m}} \sum_{n=1}^{\infty} \frac{H_{2 n}}{n^{m}}
\end{aligned}
$$

and the induction is easily completed by combining (5.8) and (5.10). Finally, using Theorem 4 and the fact that $H_{2 n}=\frac{1}{2} H_{n}+O_{n}$, we see that

$$
\sum_{n=1}^{\infty} \frac{H_{2 n}}{n^{m}}=\frac{1}{2} \zeta_{H}(m)+\zeta_{O}(m)=\mathbb{Q} \pi^{m+1}+\sum_{k=1}^{(m-1) / 2} \mathbb{Q}(\pi) J_{2 k+1,2 \pi}+\zeta_{O}(m)
$$

Gathering (5.9) and (5.11)

$$
\zeta_{O}(m)=\mathbb{Q} \pi^{m+1}+\sum_{k=1}^{(m-1) / 2} \mathbb{Q}(\pi) J_{2 k+1,2 \pi}+\sum_{k=1}^{m-1} \mathbb{Q}(\pi) J_{k, \pi}
$$

follows when $m \geq 3$ is odd. Once again the coefficients in (5.12) are all computable. Adding (3.2) to (3.2) in which $t$ has been replaced by $t+\pi$, we obtain

$$
\sum_{n=1}^{\infty}\left(\frac{H_{2 n}}{n}-\frac{1}{2 n^{2}}\right) \sin 2 n t=\frac{1}{2}(t-\pi) \log \left(2 \sin \frac{t}{2}\right)+\frac{t}{2} \log \left(2 \cos \frac{t}{2}\right)
$$

for $0<t<\pi$. We can similarly show that termwise integration of the lefthand side of (5.13) is valid. Thus for any even $m \geq 2$,

$$
\begin{aligned}
& \sum_{n=1}^{\infty}\left(\frac{H_{2 n}}{n}-\frac{1}{2 n^{2}}\right) \int_{0}^{\pi} t^{m} \sin 2 n t d t \\
& \quad=\frac{1}{2} \int_{0}^{\pi} t^{m}(t-\pi) \log \left(2 \sin \frac{t}{2}\right) d t+\frac{1}{2} \int_{0}^{\pi} t^{m+1} \log \left(2 \cos \frac{t}{2}\right) d t
\end{aligned}
$$


follows. Next using (3.12),

$$
\int_{0}^{\pi} t^{m} \sin 2 n t d t=\frac{1}{2^{m+1}} \sum_{k=1}^{m / 2}(-1)^{k} \frac{m !(2 \pi)^{m-2 k+2}}{(m-2 k+2) ! n^{2 k-1}}
$$

follows. Moreover, we have

$$
\begin{gathered}
\int_{0}^{\pi} t^{m+1} \log \left(2 \cos \frac{t}{2}\right) d t=\pi^{m+1} \int_{0}^{\pi} \log \left(2 \sin \frac{t}{2}\right) d t \\
+\sum_{k=1}^{m+1}\left(\begin{array}{c}
m+1 \\
k
\end{array}\right)(-1)^{k} \pi^{m+1-k} \int_{0}^{\pi} t^{k} \log \left(2 \sin \frac{t}{2}\right) d t .
\end{gathered}
$$

Since $0=I_{0,2 \pi}=2 I_{0, \pi}$, it follows from (5.14)-(5.16) that

$$
\sum_{k=1}^{m / 2}(-1)^{k} \frac{m !(2 \pi)^{m-2 k+2}}{2^{m+1}(m-2 k+2) !} \sum_{n=1}^{\infty}\left(\frac{H_{2 n}}{n^{2 k}}-\frac{1}{2 n^{2 k+1}}\right)=\sum_{k=1}^{m} \mathbb{Q}(\pi) I_{k, \pi}
$$

Let us show by induction that

$$
\sum_{n=1}^{\infty}\left(\frac{H_{2 n}}{n^{m}}-\frac{1}{2 n^{m+1}}\right)=\sum_{k=1}^{m} \mathbb{Q}(\pi) I_{k, \pi}
$$

when $m \geq 2$ is even. Clearly, this holds for $m=2$ by (5.17). Assuming the inductive hypothesis, we rewrite the left-hand side of (5.17) as

$$
\begin{aligned}
& \sum_{k=1}^{(m-2) / 2}(-1)^{k} \frac{m !(2 \pi)^{m-2 k+2}}{2^{m+1}(m-2 k+2) !} \sum_{r=1}^{2 k} \mathbb{Q}(\pi) I_{r, \pi} \\
& +(-1)^{\frac{m}{2}} \frac{m ! \pi^{2}}{2^{m}} \sum_{n=1}^{\infty}\left(\frac{H_{2 n}}{n^{m}}-\frac{1}{2 n^{m+1}}\right) \\
& =(-1)^{\frac{m}{2}} \frac{m ! \pi^{2}}{2^{m}} \sum_{n=1}^{\infty}\left(\frac{H_{2 n}}{n^{m}}-\frac{1}{2 n^{m+1}}\right)+\sum_{r=1}^{m-2} \mathbb{Q}(\pi) I_{r, \pi}
\end{aligned}
$$

so that the induction is completed from (5.17) and (5.19). Lastly,

$$
\sum_{n=1}^{\infty}\left(\frac{H_{2 n}}{n^{m}}-\frac{1}{2 n^{m+1}}\right)=\frac{1}{2}\left(\zeta_{H}(m)-\zeta(m+1)\right)+\zeta_{O}(m)=\sum_{k=1}^{m} \mathbb{Q}(\pi) I_{k, \pi}
$$


from (5.18) combined with Theorem 3 gives

$$
\zeta_{O}(m)=\sum_{k=1}^{m / 2} \mathbb{Q}(\pi) I_{2 k+1,2 \pi}+\sum_{k=1}^{m} \mathbb{Q}(\pi) I_{k, \pi}
$$

for even $m \geq 2$, where all of the coefficients are computable. This completes the proof of Theorem 5 .

\section{Proof of Theorem 1}

Let $f$ be a Lipschitz continuous function on $[0,2 \pi]$. The integral $I_{f, 2 \pi}$ in (1.1) is improper at 0 and $2 \pi$. Note that

$$
\int_{0}^{\pi / 3} f(t) \log \left(2 \sin \frac{t}{2}\right) d t=-\int_{0}^{\infty} f\left(2 \arcsin \frac{e^{-t}}{2}\right) \frac{t e^{-t}}{\sqrt{1-\frac{e^{-2 t}}{4}}} d t
$$

converges since $f$ is bounded. Similarly,

$$
\int_{5 \pi / 3}^{2 \pi} f(t) \log \left(2 \sin \frac{t}{2}\right) d t=\int_{0}^{\pi / 3} f(2 \pi-t) \log \left(2 \sin \frac{t}{2}\right) d t
$$

converges as well. The convergence of $I_{f, 2 \pi}$ is now a consequence of (6.1) and (6.2). For $d \geq 0$, let $P_{d}([0,2 \pi])$ be the vector space of all polynomials defined on $[0,2 \pi]$ and of degree at most $d$ with real coefficients. For any continuous function $w$ defined on $[0,2 \pi]$, define

$$
E_{d}(w):=\inf _{g \in P_{d}([0,2 \pi])}\|w-g\|
$$

where as usual

$$
\|w-g\|=\sup _{x \in[0,2 \pi]}|w(x)-g(x)| .
$$

For $\delta>0$, define the modulus of continuity of $w$ as the quantity

$$
\tau(w, \delta):=\sup _{\substack{|x-y|<\delta \\ x, y \in[0,2 \pi]}}|w(x)-w(y)|
$$

By a classical theorem of Jackson (see Chapter 14 of [31]) on the best approximation of continuous functions under $\|$.$\| by polynomials of bounded degree,$ 
we know that

$$
E_{d}(w)=O\left(\tau\left(w, \frac{1}{d}\right)\right)
$$

where the implied constant in (6.3) is absolute. For further results on approximation theory, the reader is referred to the memoir of Jackson [40]. Thus for any given integer $n \geq 3$, we may write

$$
E_{n}(f)=O\left(\tau\left(f, \frac{1}{n}\right)\right) .
$$

Since $f$ is Lipschitz, we further have

$$
\tau\left(f, \frac{1}{n}\right)=O_{f}\left(\frac{1}{n}\right)
$$

where the implied constant in (6.5) depends only on $f$ (it is essentially the Lipschitz constant of $f$ ). It follows from (6.4) and (6.5) that

$$
E_{n}(f)=O_{f}\left(\frac{1}{n}\right)
$$

Since $\mathbb{Q}$ is dense in $\mathbb{R}$, using (6.6), there exists a polynomial $P(x)=a_{0}+$ $a_{1} x+\cdots+a_{r} x^{r}$ of degree $r \leq n$ (so that $a_{r} \neq 0$ ) with rational coefficients such that

$$
\|f-P\|=\sup _{x \in[0,2 \pi]}|f(x)-P(x)| \ll_{f} \frac{1}{n} .
$$

Note that $P_{d}([0,2 \pi])$ is a finite-dimensional vector subspace of the space of all continuous functions on $[0,2 \pi]$ under the supremum metric. Therefore, $E_{d}(f)$ is attained by a polynomial of degree at most $d$. Since $f$ is not a polynomial, $E_{d}(f)>0$ follows. This means that when $n$ is large enough,

$$
\|f-P\|<E_{d}(f)
$$

holds by (6.7). Thus $r>d$ by (6.8). As $d$ is arbitrary, we see that $r \leq n$ tends to infinity with $n$. From (1.1) and (6.7), we have

$\left|I_{f, 2 \pi}-\int_{0}^{2 \pi} P(t) \log \left(2 \sin \frac{t}{2}\right) d t\right| \leq\|f-P\| \int_{0}^{2 \pi}\left|\log \left(2 \sin \frac{t}{2}\right)\right| d t \ll_{f} \frac{1}{n}$. 
Moreover,

(6.10)

$\int_{0}^{2 \pi} P(t) \log \left(2 \sin \frac{t}{2}\right) d t=\sum_{m=0}^{r} a_{m} \int_{0}^{2 \pi} t^{m} \log \left(2 \sin \frac{t}{2}\right) d t=\sum_{m=2}^{r} a_{m} I_{m, 2 \pi}$

holds since $I_{0,2 \pi}=0=I_{1,2 \pi}$ (see [27]). It is necessary to have a convenient representation of $I_{m, 2 \pi}$. To this end, using Theorem 3, we may write $I_{m+1,2 \pi}$ for odd $m \geq 1$ as a telescoping sum (using $I_{1,2 \pi}=0$ again) in the form

$$
\begin{aligned}
I_{m+1,2 \pi}= & \sum_{k=1}^{(m+1) / 2}(-1)^{k} \frac{2 m !}{(m-2 k+2) !}(2 \pi)^{m-2 k+2}\left(\zeta_{H}(2 k)-\zeta(2 k+1)\right) \\
& +\sum_{j=0}^{[m / 2]-1} \pi^{2 j+1}\left(I_{m-2 j, 2 \pi}-\pi I_{m-2 j-1,2 \pi}\right) \\
& +\pi^{2 j+2}\left(I_{m-2 j-1,2 \pi}-\pi I_{m-2 j-2,2 \pi}\right) .
\end{aligned}
$$

Again using Theorem 3, we also have

$$
\begin{aligned}
& I_{m-2 j, 2 \pi}-\pi I_{m-2 j-1,2 \pi} \\
& \quad=\sum_{k=1}^{[(m-2 j) / 2]}(-1)^{k} \frac{2(m-2 j-1) !}{(m-2 j-2 k+1) !}(2 \pi)^{m-2 j-2 k+1}\left(\zeta_{H}(2 k)-\zeta(2 k+1)\right) .
\end{aligned}
$$

Combining (6.11) and (6.12), we arrive at the formula

$$
\begin{aligned}
I_{m+1,2 \pi}= & (-1)^{\frac{m+1}{2}} 4 \pi m !\left(\zeta_{H}(m+1)-\zeta(m+2)\right) \\
& +\sum_{k=1}^{(m-1) / 2}(-1)^{k} \frac{2 m !}{(m-2 k+2) !}(2 \pi)^{m-2 k+2}\left(\zeta_{H}(2 k)-\zeta(2 k+1)\right) \\
& +\sum_{k=1}^{(m-1) / 2}(-1)^{k} C_{m, k}(2 \pi)^{m-2 k+2}\left(\zeta_{H}(2 k)-\zeta(2 k+1)\right)
\end{aligned}
$$


where

(6.14)

$$
C_{m, k}:=\sum_{j=0}^{(m-2 k-1) / 2}\left(\frac{(m-2 j-1) !}{2^{2 j}(m-2 j-2 k+1) !}+\frac{(m-2 j-2) !}{2^{2 j+1}(m-2 j-2 k) !}\right)
$$

when $m \geq 1$ is odd (with the convention that the sums over $k$ are taken to be zero if $m=1)$. It follows from (6.13) and (6.14) that

$$
I_{m+1,2 \pi}=\sum_{k=1}^{(m+1) / 2} \mathbb{Q}^{\times} \pi^{m-2 k+2}\left(\zeta_{H}(2 k)-\zeta(2 k+1)\right)
$$

when $m \geq 1$ is odd, where $\mathbb{Q}^{\times}=\mathbb{Q}-\{0\}$ (actually, the coefficients in (6.15) are in $\mathbb{Z}^{\times}=\mathbb{Z}-\{0\}$ but we will not need this). On the other hand, if $m \geq 2$ is even, then we may assemble

$$
\begin{aligned}
I_{m+1,2 \pi}= & \sum_{j=0}^{(m-2) / 2} \pi^{2 j}\left(I_{m-2 j+1,2 \pi}-\pi I_{m-2 j, 2 \pi}\right) \\
& +\pi^{2 j+1}\left(I_{m-2 j, 2 \pi}-\pi I_{m-2 j-1,2 \pi}\right)
\end{aligned}
$$

and

$$
\begin{aligned}
& I_{m-2 j+1,2 \pi}-\pi I_{m-2 j, 2 \pi} \\
& \quad=\sum_{k=1}^{(m-2 j) / 2}(-1)^{k} \frac{2(m-2 j) !}{(m-2 j-2 k+2) !}(2 \pi)^{m-2 j-2 k+2}\left(\zeta_{H}(2 k)-\zeta(2 k+1)\right)
\end{aligned}
$$

to deduce the formula

$$
I_{m+1,2 \pi}=\sum_{k=1}^{m / 2}(-1)^{k} D_{m, k}(2 \pi)^{m-2 k+2}\left(\zeta_{H}(2 k)-\zeta(2 k+1)\right)
$$

with

$$
D_{m, k}:=\sum_{j=0}^{(m-2 k) / 2}\left(\frac{(m-2 j) !}{2^{2 j-1}(m-2 j-2 k+2) !}+\frac{(m-2 j-1) !}{2^{2 j}(m-2 j-2 k+1) !}\right) .
$$


As a result of $(6.16)-(6.19)$, we have

$$
I_{m+1,2 \pi}=\sum_{k=1}^{m / 2} \mathbb{Q}^{\times} \pi^{m-2 k+2}\left(\zeta_{H}(2 k)-\zeta(2 k+1)\right)
$$

when $m \geq 2$ is even. Consequently from (6.10), (6.15) and (6.20), we see that

$$
\begin{aligned}
\int_{0}^{2 \pi} P(t) \log \left(2 \sin \frac{t}{2}\right) d t=\sum_{m=1}^{r-1} a_{m+1} I_{m+1,2 \pi} \\
=\sum_{m=1}^{r-1} a_{m+1} \sum_{k=1}^{[(m+1) / 2]} \mathbb{Q}^{\times} \pi^{m-2 k+2}\left(\zeta_{H}(2 k)-\zeta(2 k+1)\right) .
\end{aligned}
$$

Then (6.21) implies that

$$
\begin{aligned}
\int_{0}^{2 \pi} & P(t) \log \left(2 \sin \frac{t}{2}\right) d t \\
= & \sum_{k=1}^{[r / 2]}\left(\sum_{m=2 k-1}^{r-1} \mathbb{Q}^{\times} a_{m+1} \pi^{m-2 k+2}\right)\left(\zeta_{H}(2 k)-\zeta(2 k+1)\right) .
\end{aligned}
$$

Note that the coefficient of $\zeta_{H}(2 k)-\zeta(2 k+1)$ in $(6.22)$ is a rational polynomial in $\pi$. Hence, we may rewrite $(6.22)$ as

$$
\begin{aligned}
\int_{0}^{2 \pi} & P(t) \log \left(2 \sin \frac{t}{2}\right) d t \\
& =\sum_{k=1}^{[r / 2]} \mathbb{Q}\left[\pi^{s}: 1 \leq s \leq r-2 k+1\right]\left(\zeta_{H}(2 k)-\zeta(2 k+1)\right)
\end{aligned}
$$

when $r \geq 2$. If any of the coefficients on the right-hand side of (6.23) is zero, then since $\pi$ is transcendental, this would force $a_{r}=0$, a contradiction. In conclusion, all of these coefficients are nonzero. Moreover, they depend on $f$ and $n$ but not on the collection of values $\zeta_{H}(2 k)-\zeta(2 k+1), k \geq 1$. Finally, gathering (6.9) and (6.23), one obtains the desired approximation property from (1.2) when $r \geq 2$. Next assume that $f$ is a $\mathcal{C}^{\infty}$ function defined on $[0,2 \pi]$ that is not a polynomial. Then for any integer $q \geq 1, f^{(q-1)}$ is a continuously differentiable function on $[0,2 \pi]$. Using Jackson's theorem (see Chapter 14 
of [31]), we have

$$
E_{n}(f)=O_{q}\left(\frac{1}{n^{q-1}} \tau\left(f^{(q-1)}, \frac{1}{n}\right)\right)
$$

But $f^{(q-1)}$ is Lipschitz on $[0,2 \pi]$ and we see that

$$
\tau\left(f^{(q-1)}, \frac{1}{n}\right)=O_{f, q}\left(\frac{1}{n}\right) .
$$

As a result of (6.24) and (6.25), the estimate

$$
E_{n}(f)=O_{f, q}\left(\frac{1}{n^{q}}\right)
$$

holds. Consequently, using (6.26), one derives similarly the estimate

$$
\left|I_{f, 2 \pi}-\int_{0}^{2 \pi} P(t) \log \left(2 \sin \frac{t}{2}\right) d t\right| \ll_{f, q} \frac{1}{n^{q}}
$$

and the approximation

$$
\left|I_{f, 2 \pi}-\mathcal{L}\right| \ll_{f, q} \frac{1}{n^{q}}
$$

for $r \geq 2$. The coefficients in $\mathcal{L}$ are nonzero, depend on $f, n$ and $q$ but not on the collection of values $\zeta_{H}(2 k)-\zeta(2 k+1), k \geq 1$. To complete the proof, we need to show that the same approximation properties as in (6.27) hold also for any real number $\alpha$. To this end, for any given real number $\alpha$, it suffices to construct a $\mathcal{C}^{\infty}$ function $f_{\alpha}$ on $[0,2 \pi]$ corresponding to $\alpha$ that is not a polynomial and with the property that $\alpha=I_{f_{\alpha}, 2 \pi}$. Choose $\delta>0$ such that

$$
\log \left(2 \sin \frac{t}{2}\right) \geq \frac{\log 2}{2}
$$

holds for $t \in[\pi-2 \delta, \pi+2 \delta] \subseteq[0,2 \pi]$. Then consider a $\mathcal{C}^{\infty}$ function $f$ on $[0,2 \pi]$ defined as

$$
f(t)= \begin{cases}M & \text { if } t \in[\pi-\delta, \pi+\delta] \\ 0 & \text { if } t \in[0, \pi-2 \delta] \cup[\pi+2 \delta, 2 \pi],\end{cases}
$$

where $M>0$ is a constant and $0 \leq f(t) \leq M$ for all $t \in[0,2 \pi]$ (the existence and construction of such a function $f$ are of course well-known). As a result 
of (6.28) and (6.29), we have

$$
I_{f, 2 \pi} \geq M \delta \log 2
$$

Given any number $\alpha>0$, choose $M_{1}>0$ large enough such that $M_{1} \delta \log 2>$ $\alpha$. Let $f_{1}$ be the $\mathcal{C}^{\infty}$ function corresponding to $M_{1}$ and constructed similarly as $f$. Moreover,

$$
I_{f, 2 \pi} \leq 2 M \pi \log 2
$$

holds. We can find $M_{2}>0$ small enough such that $2 M_{2} \pi \log 2<\alpha$. Let $f_{2}$ be the $\mathcal{C}^{\infty}$ function corresponding to $M_{2}$. Clearly, $f_{1}$ and $f_{2}$ are not polynomials and belong to the space of $\mathcal{C}^{\infty}$ functions on $[0,2 \pi]$. Consequently, for any $0 \leq \lambda \leq 1$, the function $f_{\lambda}=\lambda f_{1}+(1-\lambda) f_{2}$ is also $\mathcal{C}^{\infty}$ on $[0,2 \pi]$ and is not a polynomial since $f_{\lambda}(t)=\lambda M_{1}+(1-\lambda) M_{2}>0$ for $t \in[\pi-\delta, \pi+\delta]$ and $f_{\lambda}(t)=0$ for $t \in[0, \pi-2 \delta] \cup[\pi+2 \delta, 2 \pi]$. Consider the real valued map $\Phi$ defined on the space of $\mathcal{C}^{\infty}$ functions on $[0,2 \pi]$ by

$$
\Phi(g)=\int_{0}^{2 \pi} g(t) \log \left(2 \sin \frac{t}{2}\right) d t
$$

for any $\mathcal{C}^{\infty}$ function $g$ on $[0,2 \pi]$. Using (6.32), we have

$$
|\Phi(g)-\Phi(h)| \leq K\|g-h\|
$$

for any two $\mathcal{C}^{\infty}$ functions $g, h$ on $[0,2 \pi]$, where

$$
K=\int_{0}^{2 \pi}\left|\log \left(2 \sin \frac{t}{2}\right)\right| d t>0 .
$$

Therefore, $\Phi$ is continuous with respect to the supremum metric on the space $\mathcal{C}^{\infty}[0,2 \pi]$. The set of functions $\mathcal{A}:=\left\{f_{\lambda}: 0 \leq \lambda \leq 1\right\}$ is a connected subset of $\mathcal{C}^{\infty}[0,2 \pi]$ since it is pathwise connected (by the very definition of $\mathcal{A})$. Thus $\Phi(\mathcal{A})$ is a connected subset of $\mathbb{R}$ containing $\Phi\left(f_{1}\right)$ and $\Phi\left(f_{2}\right)$. But because of (6.30) and (6.31), $\alpha$ is between $\Phi\left(f_{1}\right)$ and $\Phi\left(f_{2}\right)$. It follows that $\alpha \in \Phi(\mathcal{A})$ and this means that there exists a function $f_{\alpha} \in \mathcal{C}^{\infty}[0,2 \pi]$ which is not a polynomial with the property that

$$
\alpha=\int_{0}^{2 \pi} f_{\alpha}(t) \log \left(2 \sin \frac{t}{2}\right) d t .
$$


A similar argument holds for $\alpha<0$ as well by taking $-f_{\alpha}$. Lastly, observe that

$$
\int_{0}^{2 \pi} \cos \frac{t}{2} \log \left(2 \sin \frac{t}{2}\right) d t=0 .
$$

Clearly, $\cos \frac{t}{2} \in \mathcal{C}^{\infty}[0,2 \pi]$ is not a polynomial and this covers the case $\alpha=0$. Proof of Theorem 1 is now complete.

\section{Proof of Theorem 2}

The proof is similar to the proof of Theorem 1 so we mention only the changes that need to be made. If $P(x)=b_{0}+b_{1} x+\cdots+b_{r} x^{r}$ of degree $r \leq n$ with rational coefficients is the approximating polynomial of $f(x)$, then

$$
\int_{0}^{2 \pi} P(t) \log ^{2}\left(2 \sin \frac{t}{2}\right) d t=b_{0} J_{0,2 \pi}+b_{1} J_{1,2 \pi}+\sum_{m=2}^{r} b_{m} J_{m, 2 \pi}
$$

follows. We know that

$$
J_{0,2 \pi}=\frac{\pi^{3}}{6}
$$

Moreover,

$$
J_{1,2 \pi}=\frac{\pi^{4}}{6}
$$

is given in [27]. From Theorem 4, one has

$$
J_{m, 2 \pi}=\mathbb{Q}^{\times} \pi^{m+3}+\sum_{k=1}^{[m / 2]} \mathbb{Q}^{\times} \pi^{m-2 k+1} \zeta_{H}(2 k+1)
$$

for $m \geq 2$. Gathering (7.1)-(7.4), the formula

$$
\begin{aligned}
\int_{0}^{2 \pi} P(t) \log ^{2}\left(2 \sin \frac{t}{2}\right) d t= & \frac{b_{0} \pi^{3}}{6}+\frac{b_{1} \pi^{4}}{6}+\sum_{m=2}^{r} \mathbb{Q}^{\times} b_{m} \pi^{m+3} \\
& +\sum_{m=2}^{r} \sum_{k=1}^{[m / 2]} \mathbb{Q}^{\times} b_{m} \pi^{m-2 k+1} \zeta_{H}(2 k+1)
\end{aligned}
$$


is immediate. We may now take

$$
P_{r}(x)=\frac{b_{0}}{6}+\frac{b_{1} x}{6}+\sum_{m=2}^{r} \mathbb{Q}^{\times} b_{m} x^{m} \in \mathbb{Q}[x]
$$

as our polynomial of degree $r$ with coefficients independent of the collection of values $\zeta_{H}(2 k+1), k \geq 1$ so that

$$
\pi^{3} P_{r}(\pi)=\frac{b_{0} \pi^{3}}{6}+\frac{b_{1} \pi^{4}}{6}+\sum_{m=2}^{r} \mathbb{Q}^{\times} b_{m} \pi^{m+3}
$$

follows by (7.6) (note that, if necessary, the coefficients belonging to $\mathbb{Q}^{\times}$in the definition of $P_{r}(x)$ can be given explicitly using Theorem 4). If $r \geq 2$, then assembling (7.5) and (7.7), we infer that

$$
\begin{aligned}
& \int_{0}^{2 \pi} P(t) \log ^{2}\left(2 \sin \frac{t}{2}\right) d t \\
& \quad=\pi^{3} P_{r}(\pi)+\sum_{k=1}^{[r / 2]}\left(\sum_{m=2 k}^{r} \mathbb{Q}^{\times} b_{m} \pi^{m-2 k+1}\right) \zeta_{H}(2 k+1) .
\end{aligned}
$$

In (7.8), the coefficient of $\zeta_{H}(2 k+1)$ is again seen to be nonzero and of the specific form $\mathbb{Q}\left[\pi^{s}: 1 \leq s \leq r-2 k+1\right]$. These coefficients are clearly independent of the collection of values $\zeta_{H}(2 k+1), k \geq 1$. The claimed approximations of the convergent improper integral $J_{f, 2 \pi}$ follow from (7.8). To complete the proof of Theorem 2 , it suffices to construct a nonpolynomial function $g_{\alpha} \in \mathcal{C}^{\infty}[0,2 \pi]$ such that $\alpha=J_{g_{\alpha}, 2 \pi}$ for any real number $\alpha$. This can be done similarly as in the proof of Theorem 1 using

$$
\frac{1}{2} M \delta \log ^{2} 2 \leq J_{f, 2 \pi} \leq 2 M \pi \log ^{2} 2,
$$

continuity of the map $\Psi: \mathcal{C}^{\infty}[0,2 \pi] \rightarrow \mathbb{R}$ defined by

$$
\Psi(g):=\int_{0}^{2 \pi} g(t) \log ^{2}\left(2 \sin \frac{t}{2}\right) d t
$$

via

and the easily verified evaluation

$$
|\Psi(g)-\Psi(h)| \leq \frac{\pi^{3}}{6}\|g-h\|
$$

$$
\int_{0}^{2 \pi} \cos \frac{t}{2} \log ^{2}\left(2 \sin \frac{t}{2}\right) d t=0 .
$$




\section{Log-sine integrals over shorter intervals}

Log-sine integrals over shorter intervals such as $[0, \pi]$ or $\left[0, \frac{\pi}{2}\right]$ are of further interest as they are naturally related to potential energy problems of charge systems located on a half circle or a quarter circle. Nevertheless, evaluations of these integrals are sporadic rather than systematic and in many cases not complete, in contrast with the log-sine integrals over $[0,2 \pi]$. Here we demonstrate this by evaluating $I_{2, \pi}, J_{1, \pi}$ and $J_{2, \pi}$. In particular, the connection of these integrals to series involving harmonic numbers is exploited. As a bonus, we further obtain the promised evaluation of $\zeta_{O}(3)$. It turns out that our results involve polylogarithmic constants and special values of the Riemann zeta function such as $\zeta(3)$. From (3.2), we see that

$$
I_{2, \pi}=\pi I_{1, \pi}+2 \pi \sum_{n=1}^{\infty} \frac{(-1)^{n-1} H_{n}}{n^{2}}-2 \pi \sum_{n=1}^{\infty} \frac{(-1)^{n-1}}{n^{3}} .
$$

Note that

$$
\sum_{n=1}^{\infty} \frac{(-1)^{n-1}}{n^{3}}=\frac{3 \zeta(3)}{4}
$$

By a formula of De Doelder [32, Equation (5)], we have

$$
\sum_{n=1}^{\infty} \frac{(-1)^{n-1} H_{n-1}}{n^{2}}=-\frac{\zeta(3)}{8}
$$

It follows from (8.2) and (8.3) that

$$
\sum_{n=1}^{\infty} \frac{(-1)^{n-1} H_{n}}{n^{2}}=\sum_{n=1}^{\infty} \frac{(-1)^{n-1} H_{n-1}}{n^{2}}+\sum_{n=1}^{\infty} \frac{(-1)^{n-1}}{n^{3}}=\frac{5 \zeta(3)}{8}
$$

The evaluation

$$
I_{1, \pi}=\frac{7 \zeta(3)}{4}
$$

was known to Euler (see [41]). We infer from (8.1), (8.2), (8.4) and (8.5) that $I_{2, \pi}=\frac{3 \pi \zeta(3)}{2}$. Next multiplying both sides of (4.4) by $t$ and then integrating 
from 0 to $\pi$, one derives

$$
J_{1, \pi}=\frac{\pi^{4}}{16}-4 \sum_{n=1}^{\infty} \frac{H_{2 n-1}}{(2 n-1)^{3}}
$$

By another formula of De Doelder [32, Equation (8)], we have (8.7)

$$
\sum_{n=1}^{\infty} \frac{(-1)^{n-1} H_{n-1}}{n^{3}}=\frac{\pi^{4}}{48}-2 \mathrm{Li}_{4}\left(\frac{1}{2}\right)-\frac{7}{4} \zeta(3) \log 2+\frac{\pi^{2} \log 2}{12}-\frac{\log ^{4} 2}{12}
$$

where $\operatorname{Li}_{4}(z)=\sum_{n=1}^{\infty} \frac{z^{n}}{n^{4}}$ for $|z| \leq 1$ is the polylogarithmic function of order 4. As a consequence of $(8.7)$ and $\zeta(4)=\frac{\pi^{4}}{90}$, we deduce that (8.8)

$$
\sum_{n=1}^{\infty} \frac{(-1)^{n-1} H_{n}}{n^{3}}=\frac{11 \pi^{4}}{360}-2 \operatorname{Li}_{4}\left(\frac{1}{2}\right)-\frac{7}{4} \zeta(3) \log 2+\frac{\pi^{2} \log 2}{12}-\frac{\log ^{4} 2}{12}
$$

Clearly,

$$
\sum_{n=1}^{\infty} \frac{H_{2 n-1}}{(2 n-1)^{3}}=\frac{1}{2} \sum_{n=1}^{\infty} \frac{H_{n}}{n^{3}}+\frac{1}{2} \sum_{n=1}^{\infty} \frac{(-1)^{n-1} H_{n}}{n^{3}}
$$

holds. Using (8.8), (8.9) and $\zeta_{H}(3)=\frac{\pi^{4}}{72}$, one obtains

$$
\sum_{n=1}^{\infty} \frac{H_{2 n-1}}{(2 n-1)^{3}}=\frac{\pi^{4}}{45}-\mathrm{Li}_{4}\left(\frac{1}{2}\right)-\frac{7}{8} \zeta(3) \log 2+\frac{\pi^{2} \log 2}{24}-\frac{\log ^{4} 2}{24}
$$

Combining (8.6) and (8.10),

$$
J_{1, \pi}=4 \operatorname{Li}_{4}\left(\frac{1}{2}\right)+\frac{7}{2} \zeta(3) \log 2-\frac{\pi^{2} \log 2}{6}+\frac{\log ^{4} 2}{6}-\frac{19 \pi^{4}}{720}
$$

follows. Moreover, from (4.4), we have

$$
J_{2, \pi}=\frac{17 \pi^{5}}{360}-4 \pi \sum_{n=1}^{\infty} \frac{(-1)^{n-1} H_{n}}{n^{3}} .
$$

Thus by (8.8) and (8.11), the formula

$$
J_{2, \pi}=8 \pi \operatorname{Li}_{4}\left(\frac{1}{2}\right)+7 \pi \zeta(3) \log 2-\frac{\pi^{3} \log 2}{3}+\frac{\pi \log ^{4} 2}{3}-\frac{3 \pi^{5}}{40}
$$


holds. Finally, observing that

$$
\zeta_{O}(3)=8 \sum_{n=1}^{\infty} \frac{H_{2 n}}{(2 n)^{3}}-\frac{\zeta_{H}(3)}{2}=\frac{15 \zeta_{H}(3)}{2}-8 \sum_{n=1}^{\infty} \frac{H_{2 n-1}}{(2 n-1)^{3}}
$$

and using (8.10), we are led to the evaluation

$$
\zeta_{O}(3)=8 \operatorname{Li}_{4}\left(\frac{1}{2}\right)+7 \zeta(3) \log 2-\frac{\pi^{2} \log 2}{3}+\frac{\log ^{4} 2}{3}-\frac{53 \pi^{4}}{720}
$$

This completes the verification of the following result.

\section{Theorem 6.}

$$
\begin{aligned}
I_{2, \pi} & =\frac{3 \pi \zeta(3)}{2} \\
J_{1, \pi} & =4 \operatorname{Li}_{4}\left(\frac{1}{2}\right)+\frac{7}{2} \zeta(3) \log 2-\frac{\pi^{2} \log 2}{6}+\frac{\log ^{4} 2}{6}-\frac{19 \pi^{4}}{720} \\
J_{2, \pi} & =8 \pi \operatorname{Li}_{4}\left(\frac{1}{2}\right)+7 \pi \zeta(3) \log 2-\frac{\pi^{3} \log 2}{3}+\frac{\pi \log ^{4} 2}{3}-\frac{3 \pi^{5}}{40} \\
\zeta_{O}(3) & =8 \operatorname{Li}_{4}\left(\frac{1}{2}\right)+7 \zeta(3) \log 2-\frac{\pi^{2} \log 2}{3}+\frac{\log ^{4} 2}{3}-\frac{53 \pi^{4}}{720}
\end{aligned}
$$

Let us remark that the evaluations given in Theorem 6 do not seem to have further simplifications since $\mathrm{Li}_{4}\left(\frac{1}{2}\right)$ and $\zeta(3)$ cannot be written in terms of elementary constants under current knowledge. However, both of these constants have rapidly converging series representations. Specifically, we have

$$
\operatorname{Li}_{4}\left(\frac{1}{2}\right)=\sum_{n=1}^{\infty} \frac{1}{2^{n} n^{4}} \text { and } \zeta(3)=\frac{5}{2} \sum_{n=1}^{\infty} \frac{(-1)^{n-1}}{\left(\begin{array}{c}
2 n \\
n
\end{array}\right) n^{3}}
$$

where the series for $\zeta(3)$ is due to Apéry [14] and served as the starting point of his celebrated proof of the irrationality of $\zeta(3)$. Both of these series converge at a geometric rate and consequently, the formulas in Theorem 6 are suitable for numerical approximations having high accuracy.

\section{Acknowledgment}

The author is grateful to the referees for many valuable comments and suggestions that improved the presentation of the paper. 


\section{References}

[1] S. Albeverio and C. Cacciapuoti, The Riemann Zeta in terms of the dilogarithm, J. Number Theory 133 (2013), 242-277.

[2] E. Alkan, On Dirichlet L-functions with periodic coefficients and Eisenstein series, Monatsh. Math. 163 (2011), 249-280.

[3] E. Alkan, On the mean square average of special values of L-functions, J. Number Theory 131 (2011), 1470-1485.

[4] E. Alkan, Values of Dirichlet L-functions, Gauss sums and trigonometric sums, Ramanujan J. 26 (2011), 375-398.

[5] E. Alkan, On linear combinations of special values of L-functions, Manuscripta Math. 139 (2012), 473-494.

[6] E. Alkan, Averages of values of L-series, Proc. Amer. Math. Soc. 141 (2013), 1161-1175.

[7] E. Alkan, Series representations in the spirit of Ramanujan, J. Math. Anal. Appl. 410 (2014), 11-26.

[8] E. Alkan, Series representing transcendental numbers that are not $U$-numbers, submitted.

[9] E. Alkan, K. Ford, and A. Zaharescu, Diophantine approximation with arithmetic functions. I, Trans. Amer. Math. Soc. 361 (2009), 22632275 .

[10] E. Alkan, K. Ford, and A. Zaharescu, Diophantine approximation with arithmetic functions. II, Bull. Lond. Math. Soc. 41 (2009), 676-682.

[11] G.E. Andrews, R. Askey, and R. Roy, Special Functions, in 'Encyclopedia of Mathematics and its Applications', ed. G.-C. Rota, vol 71, Cambridge University Press, Cambridge, 1999.

[12] G.E. Andrews, J. Jimenez-Urroz, and K. Ono, q-series identities and values of certain L-functions, Duke Math. J. 108 (2001), 395-419.

[13] K. Aomoto, Jacobi polynomials associated with Selberg integrals, SIAM J. Math. Anal. 18 (1987), 545-549.

[14] R. Apéry, Irrationalité de $\zeta(2)$ et $\zeta(3)$, Astérisque 61 (1979), 11-13.

[15] A.T. Benjamin, D. Gaebler, and R. Gaebler, A combinatorial approach to hyperharmonic numbers, Integers 3 (2003), 1-9. 
[16] B.C. Berndt, Ramanujan's Notebooks, Part I, Springer-Verlag, New York, 1985.

[17] B.C. Berndt, Ramanujan's Notebooks, Part II, Springer-Verlag, New York, 1989.

[18] S. Bloch, Algebraic cycles and higher K-theory, Adv. Math. 61 (1986), 267-304.

[19] S. Bloch and K. Kato, L-functions and Tamagawa numbers of motives, The Grothendieck Festschrift, 1 (1990), 333-400.

[20] D. Borwein and J.M. Borwein, On an intriguing Integral and some series related to ל(4), Proc. Amer. Math. Soc. 123 (1995), 1191-1198.

[21] J.M. Borwein, D.M. Bradley, D.J. Broadhurst, and P. Lisoněk, Special values of multiple polylogarithms, Trans. Amer. Math. Soc. 353 (2001), 907-941.

[22] D.W. Boyd, Mahler's measure and special values of L-functions, Exp. Math. 7 (1998), 37-82.

[23] X.J. Chen and W. Chu, The Gauss ${ }_{2} F_{1}(1)$-summation theorem and harmonic number identities, Integral Trans. Spec. Funct. 20 (2009), 925-935.

[24] Y.J. Cho, J. Choi, M. Jung and H.M. Srivastava, Closed form evaluations of definite integrals and associated infinite series involving the Riemann zeta function, Int. J. Comput. Math. 83 (2006), 461-472.

[25] Y.J. Cho, J. Choi and H.M. Srivastava, Log-sine integrals involving series associated with the zeta function and polylogarithms, Math. Scand. 105 (2009), 199-217.

[26] J. Choi and H.M. Srivastava, Some applications of the gamma and polygamma functions involving convolutions of the Rayleigh functions, multiple Euler sums and Log-sine integrals, Math. Nachr. 282 (2009), 1709-1723.

[27] J. Choi and H.M. Srivastava, Explicit evaluations of some families of log-sine and log-cosine integrals, Integral Trans. Spec. Funct. 22 (2011), 767-783.

[28] W. Chu, Abel's lemma on summation by parts and basic hypergeometric series, Adv. Appl. Math. 39 (2007), 490-514. 
[29] W. Chu, Infinite series identities on harmonic numbers, Results Math. 61 (2012), 209-221.

[30] J.H. Conway and R.K. Guy, The Book of Numbers, Springer-Verlag, New York, 1996.

[31] K.R. Davidson and A.P. Donsig, Real analysis and applications, in 'Theory and practice (Undergraduate Texts in Mathematics)', eds. S. Axler and K. Ribet, Springer, New York, 2010.

[32] P.J. De Doelder, On some series containing $\psi(x)-\psi(y)$ and $(\psi(x)-$ $\psi(y))^{2}$ for certain values of $x$ and $y$, J. Comput. Appl. Math. 37 (1991), 125-141.

[33] F.J. Dyson, Statistical theory of the energy levels of complex systems. I, J. Math. Phys. 3 (1962), 140-156.

[34] F.J. Dyson, Statistical theory of the energy levels of complex systems. II, J. Math. Phys. 3 (1962), 157-165.

[35] F.J. Dyson, Statistical theory of the energy levels of complex systems. III, J. Math. Phys. 3 (1962), 166-175.

[36] F.J. Dyson and M.L. Mehta, Statistical theory of the energy levels of complex systems. IV, J. Math. Phys. 4 (1963), 701-712.

[37] F.J. Dyson and M.L. Mehta, Statistical theory of the energy levels of complex systems. V, J. Math. Phys. 4 (1963), 713-719.

[38] P.J. Forrester and S.O. Warnaar, The importance of the Selberg integral, Bull. Amer. Math. Soc. 45 (2008), 489-534.

[39] D.J. Griffiths, Introduction to Quantum Mechanics, 2nd Edn, Pearson, Prentice-Hall, 2005.

[40] D. Jackson, Theory of Approximation, American Mathematical Society Colloquium Publications, 11, New York, 1930.

[41] S. Kanemitsu, H. Kumagai, and M. Yoshimoto, On rapidly convergent series expressions for zeta and L-values, and Log Sine integrals, Ramanujan J. 5 (2001), 91-104.

[42] A. A. Karatsuba and S. M. Voronin, The Riemann Zeta-Function, in 'De Gruyter Expositions in Mathematics,' 5, Berlin, New York, 1992.

[43] K. Kato, A generalization of local class field theory by using $K$-groups, I. J. Fac. Sci. University of Tokyo, Sec. IA 26 (1979), 303-376. 
[44] L. Lewin, Polylogarithms and Associated Functions, Elsevier, North Holland, New York, 1981.

[45] L. Lewin (ed.), Structural Properties of Polylogarithms, Mathematical Surveys and Monographs, 37, American Mathematical Society, Providence, Rhode Island, 1991.

[46] K. Mahler, Zur Approximation der Exponentialfunktionen und des Logarithmus, I, II, J. Reine Angew. Math. 166 (1932), 118-150.

[47] M.L. Mehta, Random Matrices, 2nd Edn, Academic Press, Boston, 1991.

[48] I. Mezö and A. Dil, Hyperharmonic series involving Hurwitz zeta function, J. Number Theory 130 (2010), 360-369.

[49] Y. Ohno and W. Zudilin, Zeta stars, Commun. Number Theory Phys. 2 (2008), 325-347.

[50] M. Rapoport, N. Schappacher, and P. Schneider (eds), Beilinson's conjectures on special values of $L$-functions, in 'Perspectives in Mathematics', 4, Academic Press, Boston, 1988.

[51] M. Rogers and W. Zudilin, From L-series of elliptic curves to Mahler measures, Compos. Math. 148 (2012), 385-414.

[52] A. Selberg, Bemerkninger om et multipelt integral, Norske Mat. Tidsskr. 26 (1944), 71-78.

[53] L.-C. Shen, Remarks on some integrals and series involving the Stirling numbers and $\zeta(n)$, Trans. Amer. Math. Soc. 347 (1995), 1391-1399.

[54] A. Zaharescu and M. Zaki, An algebraic independence result for Euler products of finite degree, Proc. Amer. Math. Soc. 137 (2009), 12751283.

Department of Mathematics

KOÇ UNIVERSITY

34450, SARIYER

ISTANBUL

TURKEY

E-mail address: ealkan@ku.edu.tr

Received September 18, 2013 